IJMMS 26:1 (2001) 55-63

PII. S0161171201004112

http://ijmms.hindawi.com

(C) Hindawi Publishing Corp.

\title{
EXPERIMENT ON NUMERICAL CONFORMAL MAPPING OF UNBOUNDED MULTIPLY CONNECTED DOMAIN IN FUNDAMENTAL SOLUTIONS METHOD
}

\author{
TETSUO INOUE, HIDEO KUHARA, KANAME AMANO, and DAI OKANO
}

(Received 20 November 1999)

\begin{abstract}
We are concerned with the experiment on numerical conformal mappings. A potentially theoretical scheme in the fundamental solutions method, different from the conventional one, has been recently proposed for numerical conformal mappings of unbounded multiply connected domains. The scheme is based on the asymptotic theorem on extremal weighted polynomials. The scheme has the characteristic called "invariant and dual." Applying the scheme for typical examples, we will show that the numerical results of high accuracy may be obtained.
\end{abstract}

2000 Mathematics Subject Classification. 30E10, 41A10, 65E05.

1. Introduction. The fundamental solutions method (or charge simulation method) has been applied to the problem in electrical engineering, numerical conformal mappings [2, 3, 4, 10, 12] and Dirichlet problems [10, 16, 17].

The principle of the method is the approximation of the solution by a linear combination of logarithmic potentials. Though the method requires only solving a system of simultaneous linear equations, it is possible to get a rather precise solution for boundary problems with respect to domains bounded by smooth curves.

Amano [2, 3] has recently proposed two kinds of schemes of approximations for the conformal mappings onto the domains with circular or radial cuts, respectively.

Kuhara [11, 12] has also established a construction method of the functions mapping multiply connected domains onto the rings with circular or radial slits, based upon the works of Bergman [5] and using the fundamental solutions method. The method is described from the two-dimensional electrostatic point of view.

A potentially theoretical scheme in the fundamental solutions method, different from the conventional one, has been recently proposed for numerical conformal mappings of unbounded multiply connected domains [9]. The scheme is based on the asymptotic theorem on extremal weighted polynomials [7, 8, 13, 14, 15]. The scheme has the characteristic called "invariant and dual" with respect to interior and exterior domains [9]. In this paper, applying the scheme for typical examples, we show that the numerical results of high accuracy may be obtained.

2. Scheme for numerical conformal mapping. In this section, we show the scheme computing the approximations for the conformal mappings of "unbounded" multiply connected domains, which has been recently proposed in [9]. 
Let $D$ and $D^{\prime}$ denote unbounded multiply connected domains whose boundaries $\gamma$ and $\gamma^{\prime}$ consist of Jordan curves $\gamma_{i}$ and $\gamma_{i}^{\prime}(i=0(1) m)$, respectively.

We assume that $\gamma_{0}, \gamma_{0}^{\prime}$ enclose the origin. Let $\gamma_{0}^{\prime}$ be a circle $\left\{w:|w|=r_{0}\right\}$. Let $f(z)$ map conformally $D$ onto $D^{\prime}$ with the continuation to a bijection mapping from

$$
D \cup \gamma \text { to } D^{\prime} \cup \gamma^{\prime}
$$

corresponding $\gamma_{i}$ to $\gamma_{i}^{\prime} . f(z)$ is uniquely determined under the condition $f(\infty)=\infty$, $f^{\prime}(\infty)=1[1]$.

We have proposed in [9] the following scheme of approximations of $f(z)$

$$
f_{n}(z)=z \prod_{i=1}^{n}\left(1-\frac{z_{n, i}}{z}\right)^{\alpha_{i}}, \quad \sum_{i=1}^{n} \alpha_{i}=1,
$$

where the charge points $\left\{z_{n, i}\right\}_{i=1}^{n}$ are appropriately chosen interior to $\gamma$.

When $D^{\prime}$ is $\left\{w:|w|>r_{0}\right\}$ with radial cuts $\sum_{j=1}^{m} \gamma_{i}^{\prime}$, we propose the algorithm computing approximations of $f(z)$ as follows.

SCHEME 2.1. The approximation $f_{n}(z)$ of $f(z)$ may be obtained as follows:

(2a) $\left\{z_{n_{j}, i}^{(j)}\right\}_{i=1}^{n_{j}}$ and $\left\{\zeta_{n_{j}, i}^{(j)}\right\}_{i=1}^{n_{j}}$ with $n_{0}=\cdots=n_{m}=n$ are appropriately chosen interior to $\gamma_{j}$ and on $\gamma_{j}(j=0(1) m)$, respectively.

(2b) When $\alpha_{i}^{(j)}\left(i=0(1) n_{j}, j=0(1) m\right)$ are the solutions of a system of $(m+1)(n+1)$ simultaneous linear equations using Dirichlet-Neumann and charge conditions $[11,12]$ :

$$
\begin{gathered}
\alpha_{0}^{(0)}+\log \left|\zeta_{n_{0}, k}^{(0)}\right|+\sum_{j=0}^{m} \sum_{i=1}^{n_{j}} \alpha_{i}^{(j)} \log \left|1-\frac{z_{n_{j}, i}^{(j)}}{\zeta_{n_{0}, k}^{(0)}}\right|=0 \quad\left(k=1(1) n_{0}\right), \\
\alpha_{0}^{(l)}+\arg \left(\zeta_{n_{l}, k}^{(l)}\right)+\sum_{j=0}^{m} \sum_{i=1}^{n_{j}} \alpha_{i}^{(j)} \arg \left(1-\frac{z_{n_{j}, i}^{(j)}}{\zeta_{n_{l}, k}^{(l)}}\right)=0 \quad\left(k=1(1) n_{l}, \quad l=1(1) m\right), \\
\sum_{i=1}^{n_{0}} \alpha_{i}^{(0)}=1, \quad \sum_{i=1}^{n_{j}} \alpha_{i}^{(j)}=0 \quad(j=1(1) m),
\end{gathered}
$$

the charges at $\left\{z_{n_{j}, i}^{(j)}\right\}_{i=1}^{n_{j}}$ are given by $\left\{\alpha_{i}^{(j)}\right\}_{i=1}^{n_{j}}(j=1(1) m)$, respectively.

(2c) The approximation $f_{n}(z)$ is represented by

$$
f_{n}(z)=z \prod_{j=0}^{m} \prod_{i=1}^{n_{j}}\left(1-\frac{z_{n_{j}, i}^{(j)}}{z}\right)^{\alpha_{i}^{(j)}}, \quad \sum_{i=1}^{n_{0}} \alpha_{i}^{(0)}=1, \quad \sum_{i=1}^{n_{j}} \alpha_{i}^{(j)}=0 \quad(j=1(1) m) .
$$

Note that the approximations

$$
\alpha_{0}^{(0)} \simeq-\log r_{0}, \quad \alpha_{0}^{(j)} \simeq-\theta_{j} \quad(j=1(1) m)
$$

hold, where $\theta_{j}$ is the argument of $\gamma_{j}^{\prime}$. 
The invariant scheme of approximations has been first shown for the numerical Dirichlet problem by Murota $[16,17]$. It is physically natural and mathematically reasonable.

The solutions of a system of simultaneous linear equations in Scheme 2.1 are also invariant in the sense that the transformation $z \rightarrow a z(a>0)$ implies

$$
\begin{aligned}
& \alpha_{0}^{(0)} \longrightarrow \alpha_{0}^{(0)}+\log a, \\
& \alpha_{0}^{(j)} \longrightarrow \alpha_{0}^{(j)} \quad(j=1(1) m), \\
& \alpha_{i}^{(j)} \longrightarrow \alpha_{i}^{(j)} \quad(i=1(1) n, j=0(1) m) .
\end{aligned}
$$

3. A numerical example. We consider a function

$$
w=f(z)=z+\frac{1}{z-6} \quad\left(z=f^{-1}(w)=\frac{(7-5 \sqrt{(w-8) /(w-4)})}{(1-\sqrt{(w-8) /(w-4)})}\right)
$$

mapping $D$ onto $D^{\prime}$, where $D^{\prime}$ is $\{w:|w|>1\}$ with a radial cut

$$
\{w: 4 \leq \operatorname{Re}(w) \leq 8, \operatorname{Im}(z)=0\} .
$$

This corresponds to Dirichlet-Neumann problem and easy to check the accuracy of the approximation. We apply Scheme 2.1 to compute the approximations of $f(z)$.

The charge points interior to $\gamma_{1}$ and the collocation points on $\gamma_{1}$ are so chosen that

$$
l \exp \left(\frac{2 \pi j(i-1)}{n}\right)+6, \quad j=\sqrt{-1},(i=1(1) n)
$$

with $l=0.75,0.5,0.25$ and $l=1$, respectively.

On the other hand, the charge points interior to $\gamma_{0}$ and the collocation points on $\gamma_{0}$ are the images of the points

$$
l \exp \left(\frac{2 \pi j(i-1)}{n}\right), \quad j=\sqrt{-1},(i=1(1) n)
$$

being distributed on $\{w:|w|=0.75,0.5,0.25,1\}$ under the inverse function $f^{-1}(w)$ of $f(z)$, respectively.

For $n=13$, we solve a system of simultaneous linear equations (2.3) and obtain the approximations $f_{n}(z)$.

The accuracy of the errors is estimated by

$$
\left|f_{n}(z)-f(z)\right|
$$

at the points on $\gamma_{1}$

$$
\exp \left(\frac{2 \pi j(i-1)}{n}+\frac{\pi j}{n}\right)+6, \quad j=\sqrt{-1},(i=1(1) n)
$$

and the images of the points on $\gamma_{0}$

$$
\exp \left(\frac{2 \pi j(i-1)}{n}+\frac{\pi j}{n}\right), \quad j=\sqrt{-1},(i=1(1) n) .
$$

under the function $f^{-1}(w)$. 
By the maximum principle for the analytic functions, it is sufficient that the errors are estimated only on the boundary.

The numerical results are presented for the following cases (under a minor modification of the scheme in order to keep the continuity of the argument).

(3a) The charge distribution with $l=0.75$.

We show the charges in (2b) interior to $\gamma_{1}$ and $\gamma_{0}$, respectively as follows:

TABLE 3.1. The charge distribution with $n=13, l=0.75$ on $\gamma_{1}$.

\begin{tabular}{rrr}
\hline$-6.095578422303619 D-002$ & $5.357980215700247 D-003$ & $-5.370095750188362 D-002$ \\
\hline $2.111998739887658 D-002$ & $-2.743036534771264 D-002$ & $5.611121564384160 D-002$ \\
\hline $2.902003170269616 D-002$ & $2.902003170269579 D-002$ & $5.611121564384106 D-002$ \\
\hline$-2.743036534771176 D-002$ & $2.111998739887570 D-002$ & $-5.370095750188297 D-002$ \\
\hline $5.357980215700064 D-003$ & & \\
\hline
\end{tabular}

TABLE 3.2. The charge distribution with $n=13, l=0.75$ on $\gamma_{0}$.

\begin{tabular}{lll}
\hline $7.693733748274377 D-002$ & $7.693054597318545 D-002$ & $7.692230818952048 D-002$ \\
\hline $7.691974075923927 D-002$ & $7.691943391695984 D-002$ & $7.691958631030785 D-002$ \\
\hline $7.691971610941527 D-002$ & $7.691971610941528 D-002$ & $7.691958631030788 D-002$ \\
\hline $7.691943391695967 D-002$ & $7.691974075923903 D-002$ & $7.692230818952112 D-002$ \\
\hline $7.693054597318509 D-002$ & & \\
\hline
\end{tabular}

Note that

$$
\frac{1}{13}=7.692307692307693 D-002 \text {. }
$$

The errors on $\gamma_{1}$ and $\gamma_{0}$ are as follows:

TABLE 3.3. The errors with $n=13, l=0.75$ on $\gamma_{1}$.

\begin{tabular}{lll}
\hline $7.906455437100468 D-002$ & $7.272028918873658 D-002$ & $6.874725258323458 D-002$ \\
\hline $5.361607892270346 D-002$ & $5.209733883293302 D-002$ & $4.984156926710034 D-002$ \\
\hline $4.894182325162078 D-002$ & $4.984156926709897 D-002$ & $5.209733883293163 D-002$ \\
\hline $5.361607892270290 D-002$ & $6.874725258323204 D-002$ & $7.272028918873554 D-002$ \\
\hline $7.906455437100488 D-002$ & & \\
\hline
\end{tabular}

TABLE 3.4. The errors with $n=13, l=0.75$ on $\gamma_{0}$.

\begin{tabular}{lll}
\hline $1.917221469173348 D-003$ & $1.917212886788780 D-003$ & $1.917182701862004 D-003$ \\
\hline $1.916825188166824 D-003$ & $1.916448045831497 D-003$ & $1.916203808715182 D-003$ \\
\hline $1.916121835051632 D-003$ & $1.916203808714936 D-003$ & $1.916448045830375 D-003$ \\
\hline $1.916825188167161 D-003$ & $1.917182701862035 D-003$ & $1.917212886788246 D-003$ \\
\hline $1.917221469172982 D-003$ & & \\
\hline
\end{tabular}


Furthermore,

$$
\begin{aligned}
\alpha_{0}^{(1)}-\left(-\theta_{1}\right) & =\alpha_{0}^{(1)}-(-0)=1.741206340272162 D-003, \\
\alpha_{0}^{(0)}-\left(-\log r_{0}\right) & =\alpha_{0}^{(0)}-(-\log 1)=-1.040834085586084 D-017,
\end{aligned}
$$

which shows that (2.5) holds with high accuracy.

(3b) The charge distribution with $l=0.5$.

We show the charges in (2b) interior to $\gamma_{1}$ and $\gamma_{0}$, respectively as follows:

TABLE 3.5. The charge distribution with $n=13, l=0.5$ on $\gamma_{1}$.

\begin{tabular}{rrr}
\hline $5.676888435560761 D-002$ & $-1.336464863115225 D-001$ & $6.408202622703688 D-002$ \\
\hline$-1.158867927979212 D-001$ & $9.337154978330532 D-002$ & $-4.215724143899066 D-002$ \\
\hline $1.058525023602893 D-001$ & $1.058525023602879 D-001$ & $-4.215724143899442 D-002$ \\
\hline $9.337154978331164 D-002$ & $-1.158867927979256 D-001$ & $6.408202622703865 D-002$ \\
\hline$-1.336464863115230 D-001$ & & \\
\hline
\end{tabular}

TABLE 3.6. The charge distribution with $n=13, l=0.5$ on $\gamma_{0}$.

\begin{tabular}{lll}
\hline $7.690431354619905 D-002$ & $7.691784500736430 D-002$ & $7.692657263421879 D-002$ \\
\hline $7.692635000860265 D-002$ & $7.692605635027436 D-002$ & $7.692558594969530 D-002$ \\
\hline $7.692543327674563 D-002$ & $7.692543327674435 D-002$ & $7.692558594969630 D-002$ \\
\hline $7.692605635027493 D-002$ & $7.692635000860015 D-002$ & $7.692657263422217 D-002$ \\
\hline $7.691784500736203 D-002$ & & \\
\hline
\end{tabular}

Note that

$$
\frac{1}{13}=7.692307692307693 D-002 \text {. }
$$

The errors on $\gamma_{1}$ and $\gamma_{0}$ are as follows:

TABLE 3.7. The errors with $n=13, l=0.5$ on $\gamma_{1}$.

\begin{tabular}{lll}
\hline $1.686595817949471 D-002$ & $1.594668522932475 D-002$ & $1.426456576379659 D-002$ \\
\hline $1.245942289990706 D-002$ & $1.124520330394002 D-002$ & $1.730254947067371 D-002$ \\
\hline $1.872540245176557 D-002$ & $1.730254947067469 D-002$ & $1.124520330394140 D-002$ \\
\hline $1.245942289990679 D-002$ & $1.426456576379672 D-002$ & $1.594668522932589 D-002$ \\
\hline $1.686595817949507 D-002$ & & \\
\hline
\end{tabular}

TABLE 3.8. The errors with $n=13, l=0.5$ on $\gamma_{0}$.

\begin{tabular}{lll}
\hline $6.070869763412672 D-005$ & $6.733732155656440 D-005$ & $6.897867302145204 D-005$ \\
\hline $6.621157003193045 D-005$ & $6.260984607098040 D-005$ & $6.000919726996125 D-005$ \\
\hline $5.909032538686798 D-005$ & $6.000919726934812 D-005$ & $6.260984607020485 D-005$ \\
\hline $6.621157003170747 D-005$ & $6.897867302240548 D-005$ & $6.733732155617585 D-005$ \\
\hline $6.070869763390371 D-005$ & & \\
\hline
\end{tabular}


Furthermore,

$$
\begin{aligned}
\alpha_{0}^{(1)}-\left(-\theta_{1}\right) & =7.787235018993428 D-005, \\
\alpha_{0}^{(0)}-\left(-\log r_{0}\right) & =3.426078865054194 D-017,
\end{aligned}
$$

which shows that (2.5) holds with high accuracy.

(3c) The charge distribution with $l=0.25$.

We show the charges in (2b) interior to $\gamma_{1}$ and $\gamma_{0}$, respectively as follows:

TABLE 3.9. The charge distribution with $n=13, l=0.25$ on $\gamma_{1}$.

\begin{tabular}{rrr}
\hline$-4.896753338030436 D-002$ & $-7.545409516631382 D-002$ & $-4.502745232832742 D-002$ \\
\hline$-6.625722438826202 D-002$ & $-2.034627278488312 D-002$ & $-2.970889592876331 D-003$ \\
\hline $2.345397009507822 D-001$ & $2.345397009509353 D-001$ & $-2.970889593038040 D-003$ \\
\hline$-2.034627278472483 D-002$ & $-6.625722438839048 D-002$ & $-4.502745232824451 D-002$ \\
\hline$-7.545409516635260 D-002$ & & \\
\hline
\end{tabular}

TABLE 3.10. The charge distribution with $n=13, l=0.25$ on $\gamma_{0}$.

\begin{tabular}{lll}
\hline $7.692306130528526 D-002$ & $7.692308599892384 D-002$ & $7.692307475080235 D-002$ \\
\hline $7.692307800922398 D-002$ & $7.692307646531414 D-002$ & $7.692307723249565 D-002$ \\
\hline $7.692307689059898 D-002$ & $7.692307689069573 D-002$ & $7.692307723235956 D-002$ \\
\hline $7.692307646552272 D-002$ & $7.692307800894760 D-002$ & $7.692307475107583 D-002$ \\
\hline $7.692308599875447 D-002$ & & \\
\hline
\end{tabular}

Note that

$$
\frac{1}{13}=7.692307692307693 D-002 \text {. }
$$

The errors on $\gamma_{1}$ and $\gamma_{0}$ are as follows:

TABLE 3.11. The errors with $n=13, l=0.25$ on $\gamma_{1}$.

\begin{tabular}{lll}
\hline $2.620143221187881 D-005$ & $2.460270478748571 D-005$ & $2.165285311487771 D-005$ \\
\hline $1.774249777583574 D-005$ & $1.308661337754165 D-005$ & $7.354889113223171 D-006$ \\
\hline $2.287964484537497 D-009$ & $7.354889114253348 D-006$ & $1.308661337918933 D-005$ \\
\hline $1.774249777787493 D-005$ & $2.165285311546687 D-005$ & $2.460270478816393 D-005$ \\
\hline $2.620143221202827 D-005$ & & \\
\hline
\end{tabular}

TABLE 3.12. The errors with $n=13, l=0.25$ on $\gamma_{0}$.

\begin{tabular}{lll}
\hline $4.903447136123618 D-010$ & $1.019052540368634 D-009$ & $9.883685502913276 D-010$ \\
\hline $7.553265530481330 D-010$ & $4.968949958792792 D-010$ & $2.547095062921054 D-010$ \\
\hline $8.554679187256638 D-011$ & $2.547094246284032 D-010$ & $4.968949414229016 D-010$ \\
\hline $7.553265708226896 D-010$ & $9.883683875455573 D-010$ & $1.019053538929026 D-009$ \\
\hline $4.903451511212511 D-010$ & & \\
\hline
\end{tabular}


Furthermore,

$$
\begin{aligned}
\alpha_{0}^{(1)}-\left(-\theta_{1}\right) & =2.378106242066085 D-009, \\
\alpha_{0}^{(0)}-\left(-\log r_{0}\right) & =8.673617379884036 D-019,
\end{aligned}
$$

which shows that (2.5) holds with high accuracy.

The numerical example shows:

(3d) The data present the distribution of the charges and errors of the approximations, which are symmetric with respect to the real axis and with high accuracy.

(3e) When the charges and collocation points are distributed uniformly (see the definition in [6]) as (3.4), the example shows that the approximations

$$
\alpha_{i} \simeq \frac{1}{n}(i=1,2, \ldots, n), \quad \alpha_{0}^{(0)} \simeq-\log r_{0}, \quad f(z) \simeq f_{n}(z)
$$

would hold with high accuracy.

We have examined the case of odd $n$. The case of $n=12$ is shown in the following (3f).

(3f) The charge distribution with $l=0.25$.

We show the charges in (2b) interior to $\gamma_{1}$ and $\gamma_{0}$, respectively as follows:

TABLE 3.13. The charge distribution with $n=12, l=0.25$ on $\gamma_{1}$.

\begin{tabular}{rrr}
\hline$-1.942606947010962 D-001$ & $5.972416806033705 D-002$ & $-1.896074309191883 D-001$ \\
\hline $7.405835693044212 D-002$ & $-1.569787980380907 D-001$ & $2.128037039665000 D-001$ \\
\hline $1.942606947011143 D-001$ & $2.128037039664623 D-001$ & $-1.569787980380575 D-001$ \\
\hline $7.405835693042352 D-002$ & $-1.896074309191738 D-001$ & $5.972416806032727 D-002$ \\
\hline
\end{tabular}

TABLE 3.14. The charge distribution with $n=12, l=0.25$ on $\gamma_{0}$.

\begin{tabular}{lll}
\hline $8.333347749250593 D-002$ & $8.333322686829960 D-002$ & $8.333337977798844 D-002$ \\
\hline $8.333331344289655 D-002$ & $8.333334554423848 D-002$ & $8.333332530161311 D-002$ \\
\hline $8.333334063736710 D-002$ & $8.333332530170494 D-002$ & $8.333334554409336 D-002$ \\
\hline $8.333331344306075 D-002$ & $8.333337977783503 D-002$ & $8.333322686839680 D-002$ \\
\hline
\end{tabular}

Note that

$$
\frac{1}{12}=8.333333333333333 D-002 .
$$

The errors on $\gamma_{1}$ and $\gamma_{0}$ are as follows:

TABLE 3.15. The errors with $n=12, l=0.25$ on $\gamma_{1}$.

\begin{tabular}{lll}
\hline $4.889879193915697 D-004$ & $4.566691529354238 D-004$ & $4.003418633466522 D-004$ \\
\hline $3.341897580605150 D-004$ & $2.746913382174855 D-004$ & $2.377726507971854 D-004$ \\
\hline $2.377726507949406 D-004$ & $2.746913382176728 D-004$ & $3.341897580660071 D-004$ \\
\hline $4.003418633444508 D-004$ & $4.566691529379670 D-004$ & $4.889879193896632 D-004$ \\
\hline
\end{tabular}


Furthermore,

$$
\begin{aligned}
\alpha_{0}^{(1)}-\left(-\theta_{1}\right) & =8.050910065412068 D-009, \\
\alpha_{0}^{(0)}-\left(-\log r_{0}\right) & =1.001802807376606 D-016,
\end{aligned}
$$

which shows that (2.5) holds with high accuracy.

TABLE 3.16. The errors with $n=12, l=0.25$ on $\gamma_{0}$.

\begin{tabular}{lll}
\hline $2.472695830800364 D-009$ & $2.547021968349248 D-009$ & $1.880133758085106 D-009$ \\
\hline $1.954951566801633 D-009$ & $1.938261615460128 D-009$ & $1.892427103310218 D-009$ \\
\hline $1.892426551711589 D-009$ & $1.938261234766441 D-009$ & $1.954951344522423 D-009$ \\
\hline $1.880133600747165 D-009$ & $2.547022633553721 D-009$ & $2.472696052933940 D-009$ \\
\hline
\end{tabular}

We have shown the data exactly for the convenience of the readers in order to follow the numerical experiment in Fortran 90 with double precision.

The numerical calculation has been performed in MsDevf90 (PC9821-NEC).

ACKNOWLEDGEment. The authors express their gratitude to T. Fujii of Kobe Mercantile Marine College for helping to make tables.

4. Concluding remark. The numerical scheme of approximations in this paper has been applied for a doubly connected domains applying the charge simulation method.

The numerical experiments for other doubly connected domains and $m(\neq 2)$ multiply connected ones will be furthermore studied in a future paper.

\section{REFERENCES}

[1] L. V. Ahlfors, Complex Analysis: an Introduction of the Theory of Analytic Functions of one Complex Variable, 2nd ed., McGraw-Hill Book Co., New York, 1966. MR 32\#5844. Zbl 154.31904.

[2] K. Amano, Numerical conformal mapping onto radial slit domains by the charge simulation method, Sūrikaisekikenkyūsho Kōkyūroku (1995), no. 915, 107-118 (Japanese). CMP 1385 269. Zbl 941.65509.

[3] _ Numerical conformal mapping onto the circular slit domains, Trans. Inform. Process. Soc. Japan 36 (1995), no. 2, 219-225 (Japanese). CMP 1322479.

[4] A charge simulation method for numerical conformal mapping onto circular and radial slit domains, SIAM J. Sci. Comput. 19 (1998), no. 4, 1169-1187 (electronic). MR 99b:30007. Zbl 907.30009.

[5] S. Bergman, The Kernel Function and Conformal Mapping, Mathematical Surveys, no. 5, American Mathematical Society, New York, 1950. MR 12,402a. Zbl 040.19001.

[6] D. Gaier, Lectures on Complex Approximation, Birkhäuser Boston, Massachusetts, 1987. MR 88i:30059b. Zbl 612.30003.

[7] T. Inoue, Asymptotic behavior of extremal weighted polynomials, Mathematica (Cluj) 35(58) (1993), no. 1, 29-34. MR 95k:30010. Zbl 803.30005.

[8]__ Applications of asymptotic theorem on weighted extremal polynomials, Trans. Japan Soc. Ind. App. Math. 4 (1994), 151-155 (Japanese).

[9] T. Inoue, H. Kuhara, K. Amano, and D. Okano, Theoretical scheme on numerical conformal mapping of unbounded multiply connected domain by fundamental solutions method, Int. J. Math. Math. Sci. 24 (2000), no. 2, 129-137. MR 2001e:65044. Zbl 991.73479. 
[10] M. Katsurada and H. Okamoto, The collocation points of the fundamental solution method for the potential problem, Comput. Math. Appl. 31 (1996), no. 1, 123-137. CMP 1362 387. Zbl 852.65101.

[11] H. Kuhara, S. Oshima, and K. Yasuda, A charge simulation method for canonical conformal mapping of multiply connected regions onto rings with radial slits, Paper of Technical Meeting on Electromagnetic Theory, IEE and IEIC of Japan, 1997, EMT97-83.

[12] H. Kuhara, T. Yonezawa, and S. Oshima, Constructions of transformation mapping multiply connected regions conformally onto the canonical ones using the charge simulation method, Paper of Technical Meeting on Electromagnetic Theory, IEE and IEIC of Japan, 1996, EMT-96-110.

[13] H. N. Mhaskar, Finite-infinite-range inequalities in the complex plane, Int. J. Math. Math. Sci. 14 (1991), no. 4, 625-638. MR 93b:26023. Zbl 774.30004.

[14] H. N. Mhaskar and E. B. Saff, Where does the sup norm of a weighted polynomial live? (A generalization of incomplete polynomials), Constr. Approx. 1 (1985), no. 1, 71-91. MR 86a:41004. Zbl 582.41009.

[15] W Weighted analogues of capacity, transfinite diameter, and Chebyshev constant, Constr. Approx. 8 (1992), no. 1, 105-124. MR 93a:31004. Zbl 747.31001.

[16] K. Murota, On "invariance" of schemes in the fundamental solution method, Trans. Inform. Process. Soc. Japan 3 (1993), 533-535 (Japanese).

[17] Comparison of conventional and "invariant" schemes of fundamental solutions method for annular domains, Japan J. Indust. Appl. Math. 12 (1995), no. 1, 61-85. MR 95k:65098. Zbl 831.65118.

Tetsuo Inoue: DePartment of ApPlied Mathematics, Kobe MerCantile Marine College, HIGASHINADAKU, 658-0022, KOBE, JAPAN

Hideo Kuhara: Yatsusiro National College of Technology, Kumamoto, JaPAN

Kaname Amano and Dai OKano: Department of Computer Science, Faculty of EngINEERING, EHIME UNIVERSITY, EHIME, JAPAN 


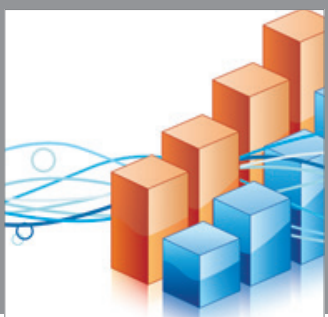

Advances in

Operations Research

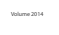

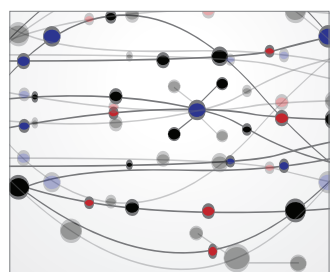

\section{The Scientific} World Journal
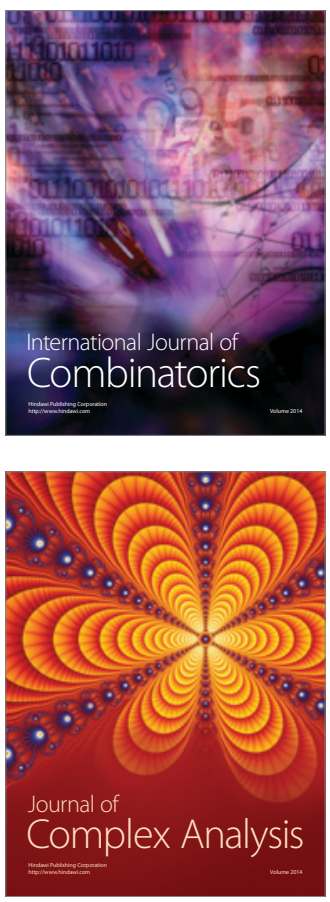

International Journal of

Mathematics and

Mathematical

Sciences
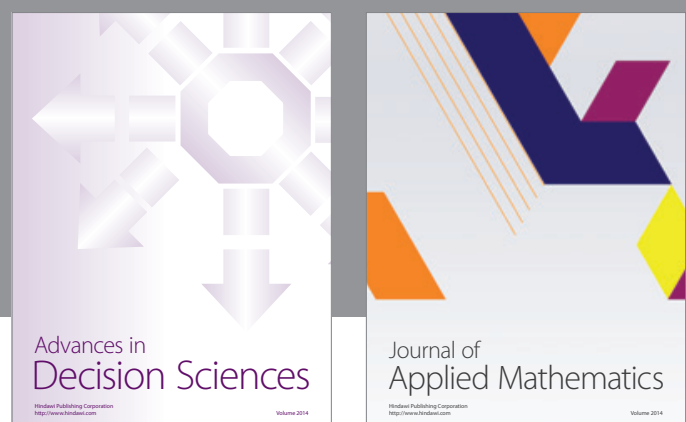

Journal of

Applied Mathematics
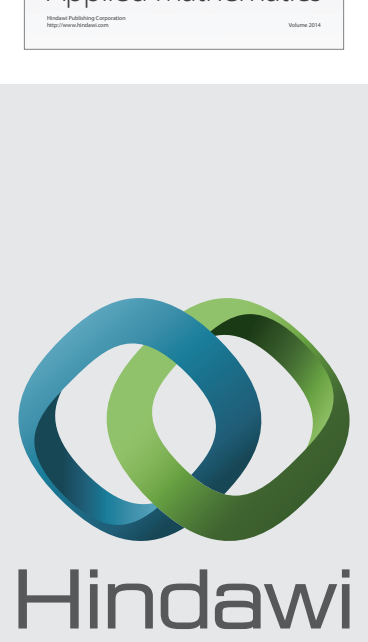

Submit your manuscripts at http://www.hindawi.com
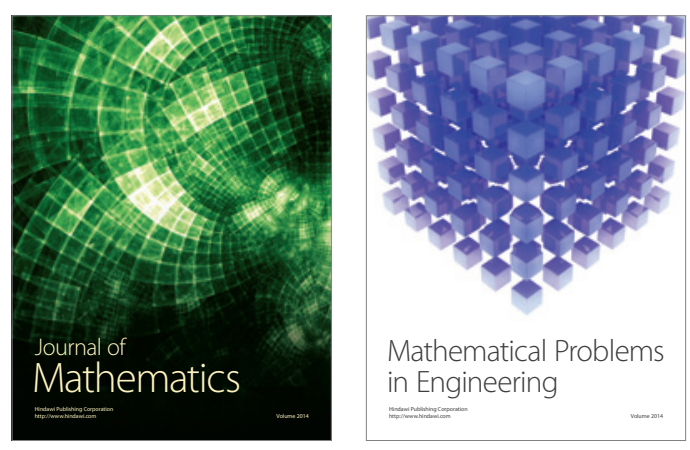

Mathematical Problems in Engineering
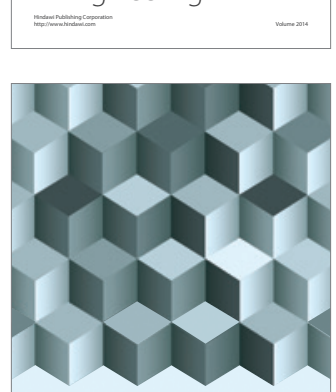

Journal of

Function Spaces
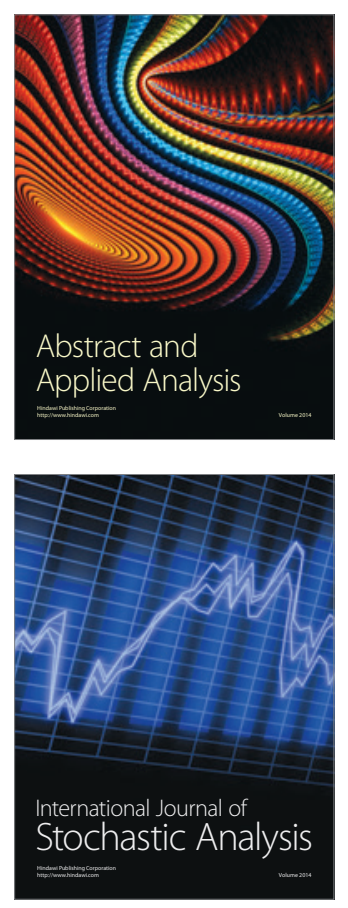

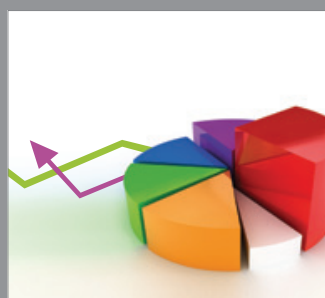

ournal of

Probability and Statistics

Promensencen
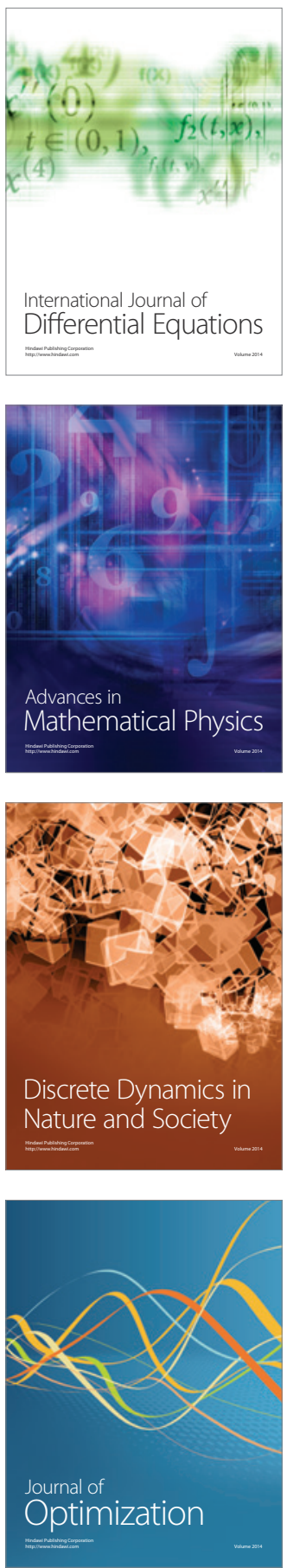